\title{
Signatures of Majorana fermions in hybrid normal-superconducting rings
}

\author{
Ph. Jacquod ${ }^{1,2,3}$ and M. Büttiker ${ }^{4, *}$ \\ ${ }^{1}$ Department of Physics, University of Arizona, Tucson, Arizona 85721, USA \\ ${ }^{2}$ College of Optical Sciences, University of Arizona, Tucson, Arizona 85721, USA \\ ${ }^{3}$ University of Applied Sciences of Western Switzerland, School of Engineering, Route du Rawyl 47, 1951 Sion, Switzerland \\ ${ }^{4}$ Department of Theoretical Physics, University of Geneva, 1211 Geneva, Switzerland \\ (Received 26 June 2013; revised manuscript received 10 December 2013; published 23 December 2013)
}

\begin{abstract}
We investigate persistent currents in metallic rings interrupted by a Coulomb-blockaded topological superconducting segment. We show that the presence of Majorana bound states in the superconductor is reflected in the emergence of an $h / e$ harmonic in the persistent current. The Majorana bound states further render the current finite at zero flux, with a sign that is determined by the fermion parity of the superconductor. The resulting peculiar symmetry of the persistent current is compatible with a free energy that is even in time-reversal symmetry-breaking fields. These unique features of the persistent currents are robust against disorder and provide unambiguous signatures of the presence of Majorana fermions.
\end{abstract}

Several recent experiments have reported features in transport $^{1-5}$ and Josephson spectroscopy ${ }^{6}$ that have varying degrees of consistency with the presence of Majorana states. ${ }^{7}$ The experimental setups are all based on the nanowire implementation of Kitaev's chain ${ }^{8}$ proposed in Refs. 9 and 10. The presence of Majorana fermions in such systems manifests itself in a zero-bias peak in the tunneling conductance into the nanowire ${ }^{11,12}$ and by a doubling of the periodicity of the Josephson current in the superconducting phase difference. ${ }^{8,13,14}$ These features were observed in Refs. 1-6. Other signatures of Majorana states in transport interferometry have also been theoretically investigated. ${ }^{12,15-17}$

Motivated by the experimental reports, a number of theoretical works have pointed out that zero-bias peaks in the tunneling conductance may also occur in the topologically trivial phase. ${ }^{18-21}$ Their observation is therefore not sufficient to demonstrate the presence of a Majorana state. Moreover, Refs. 22 and 23 showed that a doubling of the periodicity of the Josephson current as reported in the ac Josephson setup of Ref. 6 may also occur due to Landau-Zener transitions between standard Andreev bound states. Despite a slowly growing body of evidence in favor of the presence of Majorana states in nanowires, an incontrovertible experiment is still missing. The consensus at this point is that only a zero-bias tunneling conductance peak quantized at $2 e^{2} / h$ would unambiguously reflect the presence of a Majorana state. The observation of such a quantized peak would however require ideal circumstances, in particular ultralow temperatures beyond the reach of currently existing devices. ${ }^{24}$

In this paper, we propose an altogether new experiment to detect Majorana fermions in the nanowire platforms of Refs. 9 and 10. The system we investigate is sketched in Fig. 1. It consists of a normal metallic ring interrupted by a superconducting segment of length $L \gg \xi$, much larger than the superconducting coherence length $\xi$. The superconducting segment can be either in a topologically trivial state, with a superconducting gap which allows the transfer of Cooper pairs only, or in a topologically nontrivial state, with Majorana subgap states at each of its ends allowing the coherent transfer of single quasiparticles. ${ }^{25,26}$ A persistent current is triggered by a magnetic flux $\phi$ piercing the ring. In the former case, the current has even harmonics only $\sim \sin \left(4 \pi n \phi / \phi_{0}\right)$, with the flux quantum $\phi_{0}=h / e$ and $n=1,2, \ldots$ In other words, only $h / 2 e$ harmonics exist because only Cooper pairs with charge $2 e$ can be transferred through the superconductor. In the latter case, the transfer of a single electron generates odd harmonics $\sim \sin \left[2 \pi(2 n+1) \phi / \phi_{0}-\chi\right]$. The presence of Majorana states breaks time-reversal symmetry because the quasiparticle transfer amplitude $\tau$ through the topological superconductor picks a relative minus sign when the direction of transfer is inverted. This follows directly from the fermionic anticommutation relations of Majorana creation and annihilation operators. ${ }^{25,26}$ Additionally, $\tau \sim(-1)^{n_{0}}$ depends on the number $n_{0}$ of fermions on the superconductor. This leads to $\chi=(-1)^{n_{0}} \pi / 2$ and when the fermion parity is fixed, persistent current harmonics $\sim(-1)^{n_{0}} \cos \left[2 \pi(2 n+1) \phi / \phi_{0}\right]$ are obtained in the weak-coupling limit, while the odd harmonics vanish identically when the fermion parity is not fixed and an average over $n_{0}$ is taken. Building on Ref. 26, we propose to fix the fermion parity via Coulomb blockade of the superconducting segment. This in particular ensures that electron-to-hole transfers across the topological superconductor are suppressed. Additionally, hybridization between the two Majorana bound states via the normal metallic ring can be neglected because Coulomb blockade requires a weak coupling between the topological superconductor and the normal metallic ring. This justifies the use of the effective Hamiltonian derived in Ref. 26. The on-resonance persistent current then bears three unambiguous signatures of the presence of Majorana states: (i) The current develops an $h / e$ harmonic, (ii) the current is finite at zero flux in the nontrivial phase but vanishes in the trivial phase, and (iii) the current changes sign when the fermion number parity on the superconductor is changed from $n_{0}=2 n$ (unoccupied Majorana states) to $n_{0}=2 n+1$ (occupied Majorana states). We also show that despite the finite zero-field current, the system's free energy is even in time-reversal symmetrybreaking fields $\mathcal{F}\left(\phi, B_{\mathrm{Z}}\right)=\mathcal{F}\left(-\phi,-B_{\mathrm{Z}}\right)$.

The origin of the $h / e$ harmonics is the same as that of the fractional Josephson effect, however the effect is more robust for persistent currents than for the ac Josephson effect $;{ }^{6}$ the former in particular are immune to Landau-Zener 


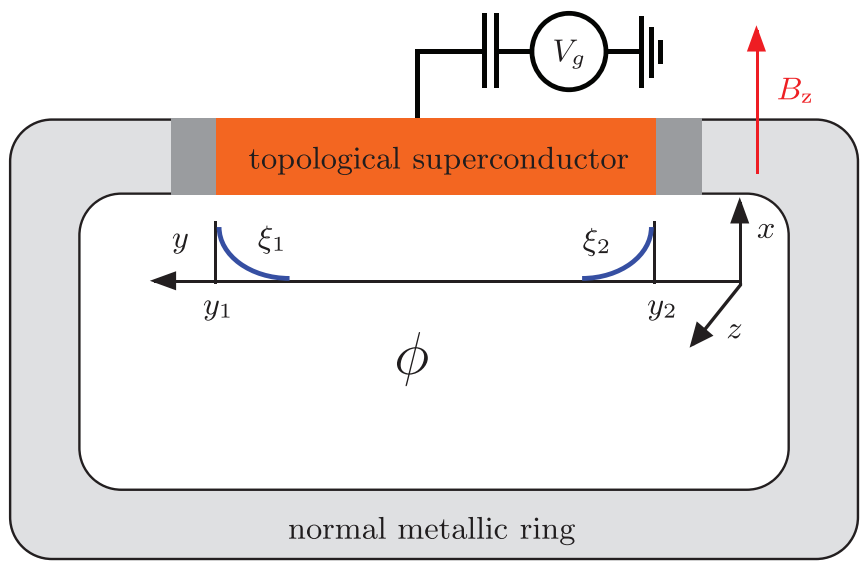

FIG. 1. (Color online) Setup to detect signatures of Majorana fermions in persistent currents through metallic rings. A spin-orbit coupled metallic nanowire (orange) with induced superconductivity is embedded in a metallic ring pierced by a magnetic flux $\phi$. A sufficiently strong Zeeman field $B_{\mathrm{Z}}$ applied perpendicular to the axis of the nanowire creates two Majorana states $\xi_{1,2}$ localized at each end of the nanowire (Refs. 9 and 10). In the presence of such states, individual electrons can be coherently transferred across the nanowire, even when the latter is longer than the superconducting coherence length, thereby generating an $h / e$ harmonic in the persistent current. The nanowire is Coulomb blockaded and tunnel coupled to the metallic ring (dark gray rectangles represent tunnel barriers). Its occupation number $n_{0}$ can be externally tuned by a gate voltage $V_{g}$, which fixes the fermion parity and allows one to change the sign of the persistent current $I(\phi=0) \propto(-1)^{n_{0}}$.

transitions..$^{23}$ Measuring persistent currents in metallic rings is challenging but has been done by several groups. ${ }^{27-31} \mathrm{In}$ persistent currents the emergence of an $h / e$ harmonic may also occur when $\xi$ increases and becomes comparable to $L, 32$ which however can be monitored experimentally. Anomalous supercurrents (a nonzero persistent current at zero phase difference) in Josephson junctions formed of a mesoscopic conductor sandwiched between two ordinary superconductors are discussed in several theoretical works (see, e.g., Refs. 33-35), where it is a consequence of the spin-orbit interaction and Zeeman fields in the normal conductor. In contrast, in our work spin-orbit interaction and Zeeman fields exist only in the superconductor to the extent that they are needed to drive the latter into the topological phase.

We calculate the canonical persistent current using the effective Hamiltonian derived by Fu (Ref. 26) for fixed-parity Coulomb-blockaded topological superconductors

$$
\begin{aligned}
H= & H_{\text {ring }}+\delta\left(f^{\dagger} f-1 / 2\right)+\left(\lambda_{1} c_{\mathrm{L}}^{\dagger} f+\text { H.c. }\right) \\
& +\left[-i \lambda_{2}(-1)^{f^{\dagger} f} c_{\mathrm{R}}^{\dagger} f \exp (i \varphi)+\text { H.c. }\right] .
\end{aligned}
$$

The superconductor is connected to a metallic ring with Hamiltonian $H_{\text {ring }}=\sum_{k} \epsilon_{k} c_{k}^{\dagger} c_{k}$, pierced by a magnetic flux $\phi=\hbar \varphi / e$; the fermionic operators $c$ annihilate an electron in the ring and $f$ is a fermion operator on the superconductor, combining Majorana operators and fermion parity isospin operators. The superconductor is Coulomb blockaded and coupled to the ring via the tunnel barrier $\lambda_{1,2} \ll t$, which allows one to project the Hamiltonian into the subspace with only two superconductor charge states $\left|n_{0}\right\rangle$ and $\left|n_{0}+1\right\rangle$. The energy difference between these two states is $\delta$, which can be tuned by an external gate voltage. Details of the construction of $H$ are given in Ref. 26. The above Hamiltonian is appropriate to calculate the $h / e$ harmonics of the persistent current close to zero chemical potential in the ring in the limit of a long superconducting segment. We will comment on the short segment limit below.

In the limit when the superconductor-ring coupling is weak, and close to resonance between $\left|n_{0}\right\rangle$ and $\left|n_{0}+1\right\rangle$ (i.e., close to $\delta=0$ ) with $M+n_{0}$ electrons in total, $H$ can be reduced to a $2 \times 2$ Hamiltonian (see Ref. 36 for a similar treatment of a metallic quantum dot embedded in a metallic ring)

$$
H_{\text {red }}=\left(\begin{array}{cc}
\epsilon_{M} & \tilde{\lambda}_{1}-i \tilde{\lambda}_{2}(-1)^{n_{0}} e^{i \varphi} \\
\tilde{\lambda}_{1}+i \tilde{\lambda}_{2}(-1)^{n_{0}} e^{-i \varphi} & \delta
\end{array}\right),
$$

where $\epsilon_{M}$ is the last occupied state in the ring when there are $n_{0}$ electrons on the superconductor. The total energy is given by the eigenvalues of $H_{\text {red }}$ plus a constant sum over all energy levels in the ring $\sum_{k=1}^{M-1} \epsilon_{k}$. The eigenvalues $E_{ \pm}(\varphi)$ of $H_{\text {red }}$ are easily calculated and one obtains the canonical persistent current $I(\varphi)=-(e / \hbar) \partial_{\varphi} E_{-}$as

$$
I(\varphi)=\frac{e}{\hbar} \frac{(-1)^{n_{0}} \tilde{\lambda}_{1} \tilde{\lambda}_{2} \cos \varphi}{\sqrt{\left(\epsilon_{M}-\delta\right)^{2} / 4+\tilde{\lambda}_{1}^{2}+\tilde{\lambda}_{2}^{2}+2 \tilde{\lambda}_{1} \tilde{\lambda}_{2}(-1)^{n_{0}} \sin \varphi}} .
$$

The $h / e$ harmonic of the current depends on the fermion parity; it is finite at zero flux and its magnitude is algebraically reduced away from resonance $\left(\delta \neq \epsilon_{M}\right)$. The weak-coupling condition means that $\tilde{\lambda} \ll \delta \epsilon$ with the energy level spacing $\delta \epsilon$ in the ring, so unless $\epsilon_{M}$ is anomalously close to zero, the $\tilde{\lambda}$ terms under the square root in the denominator are negligible at the degeneracy point between the two superconducting states, $\delta=0$. Generically, $\min \left(\left|\epsilon_{M}\right|\right) \simeq \delta \epsilon$. Further specifying to a weakly disordered $N$-site one-dimensional tight-binding ring with nearest-neighbor hopping amplitude $t$, we obtain $\tilde{\lambda}_{i}=$ $(\pi / 2 N)^{1 / 2} \lambda_{i}, \delta \epsilon \simeq \pi t / N$ for a half-filled ring, so that $I(\varphi)=$ $(e / h)(-1)^{n_{0}} \lambda_{1} \lambda_{2} \cos \varphi / t$. In the weak-coupling limit, the sign of the current at zero flux is thus determined by the fermion parity, which can be tuned via the gate voltage. The change in sign is a signature of the Friedel sum rule. ${ }^{37}$ In this weak-coupling limit, the current does not depend on the length of the ring. Alternatively, one may tune the gate voltage and work at $\epsilon_{M}=$ $\delta$, in which case the current becomes (for $\lambda_{1,2}=\lambda$ ) $I(\varphi)=$ $(e / \hbar)(-1)^{n_{0}} \pi^{1 / 2} \lambda \cos \varphi / \sqrt{4 N\left[1+(-1)^{n_{0}} \sin \varphi\right]}$. In this case, the current decays as $N^{-1 / 2}$ with the size of the ring. One important consequence of Eq. (3) is that upon disorder averaging, the $h / e$ harmonic vanishes identically because $I_{n_{0}}+I_{n_{0}+1}$ is $h / 2 e$ periodic.

There are two main differences between our Eq. (3) and Eq. (7) of Ref. 36 for a ring interrupted by a normal metallic quantum dot. The first one is that the presence of Majorana states breaks time-reversal symmetry in such a way that $\varphi \rightarrow \varphi \pm \pi / 2$, thereby turning sines into cosines and vice versa. The second is that in Eq. (3), the parity of the current is related to the fermion parity on the superconductor when the latter is in the nontrivial phase. In the case of a purely onedimensional ring, the parity of the persistent current depends 


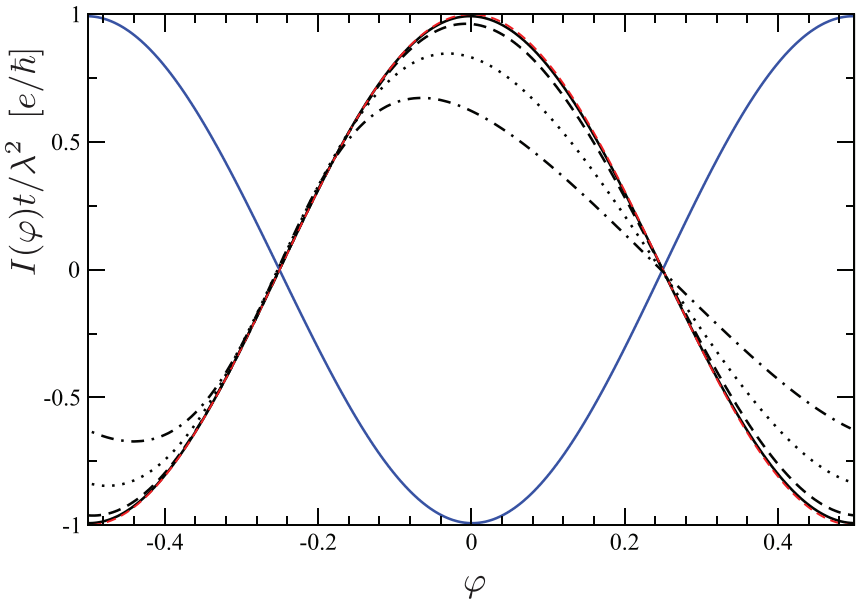

FIG. 2. (Color online) Persistent currents in the setup of Fig. 1 with weak coupling $\lambda_{1}=\lambda_{2}=0.02 t$ between the topological superconductor and the metallic ring. The metallic ring has $N=20$ (solid lines), 100 (dashed line), 500 (dotted line), and 1500 (dot-dashed line) sites. The fermion parity $n_{0}$ is even (black lines) and odd (blue line). The red dashed line gives the theoretical prediction $I(\varphi)=(e / \hbar)(-1)^{n_{0}} \lambda_{1} \lambda_{2} \cos \varphi$ (see the text). Deviations appear for larger rings with smaller level spacing for which $\tilde{\lambda}_{i}$ is no longer negligible against $\delta \epsilon$.

on the total parity of the one-dimensional ring, including both the Coulomb-blockaded segment and the metallic ring. ${ }^{36}$ This remains true also in the case of a superconducting ring because the occupancy of the metallic ring determines the relative sign of the hoppings $\lambda_{1}$ and $\lambda_{2}$. Parity effects disappear in nonstrictly one-dimensional metallic rings, however, the dependence on the fermion parity of the superconducting segment persists, even when the metallic ring carries more than one transverse channel.

To check these results and extend them beyond the weakcoupling limit we numerically calculate $I(\varphi)$ for a tightbinding version of Eq. (1) with $H_{\text {ring }}=-t \sum_{\langle i ; j\rangle}\left(c_{i}^{\dagger} c_{j}+\right.$ H.c. $)$ and $c_{\mathrm{L}}=c_{1}, c_{\mathrm{R}}=c_{N}$. We first show in Fig. 2 results for the weak-coupling regime with $\lambda_{i} \ll \delta \epsilon$. As predicted, the persistent current exhibits a simple $\cos \varphi$ behavior, with the sign determined by the fermion parity as $(-1)^{n_{0}}$. Upon increasing the size of the ring, the level spacing $\delta \epsilon$ decreases and therefore the $\lambda$ terms in the denominator of Eq. (3) are no longer entirely negligible against the $\left(\epsilon_{M}-\delta\right)^{2}$ term. This generates deviations from the pure $\cos \varphi$ behavior (dashed, dotted, and dot-dashed curve). Note that for clean one-dimensional rings with an odd number of sites, one eigenenergy vanishes, in which case the $\lambda$ terms are not negligible at resonance, $\delta=0$. We checked, but do not show, that this anomalous even-odd effect generically disappears when $\delta$ is tuned slightly away from resonance, when some disorder is added to the ring or when the latter becomes quasi-one-dimensional, with few transverse channels.

Data at stronger couplings $\lambda_{1}=\lambda_{2}=0.2 t$ are shown in Fig. 3. The persistent currents acquire a more complicated harmonic content, which is qualitatively captured by Eq. (3). Some deviations are seen, which we attribute to the fact that more than a single level is coupled to the superconductor in this regime, while Eq. (3) assumes a single-level hybridization.

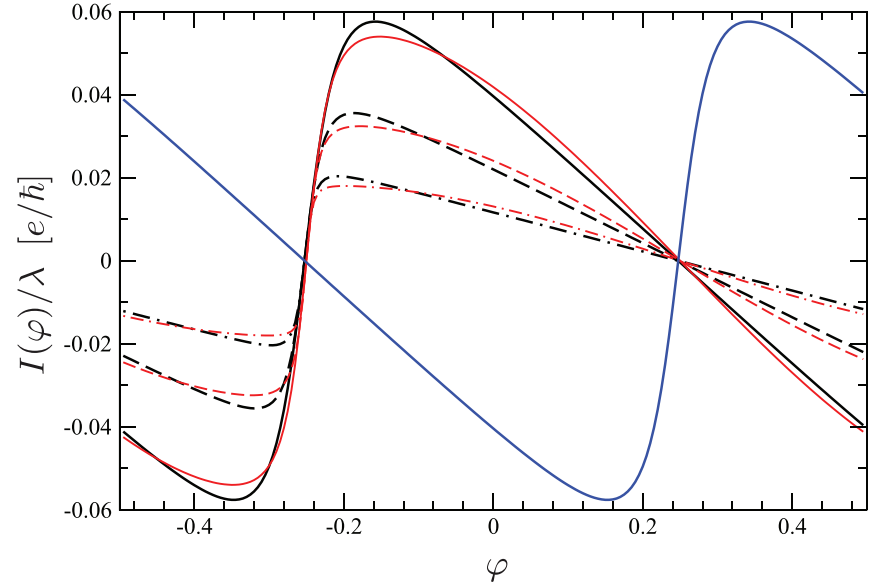

FIG. 3. (Color online) Persistent currents in the setup of Fig. 1 with larger coupling $\lambda_{1}=\lambda_{2}=0.2 t$ between the topological superconductor and the metallic ring (black and blue lines). The metallic ring has $N=100$ (solid line), 200 (dashed line), and 400 (dot-dashed line) sites, with even (black lines) and odd (blue line, for $N=100$ ) fermion parity. The red lines are best fits with Eq. (3).

For the same reason, the best fits with Eq. (3) shown have parameters that differ from the theoretical predictions. It is still remarkable that the single-level prediction of Eq. (3) captures the current symmetry $I\left(n_{0}, \varphi\right)=-I\left(n_{0}+1,-\varphi\right)$, even when more than one ring level is coupled to the Majorana states.

Our numerics confirm our theoretical prediction that in clean, one-dimensional metallic rings interrupted by a Coulomb-blockaded superconducting segment carrying Majorana bound states, persistent currents have $h / e$ periodicity and finite magnitude even when no flux pierces the ring and furthermore change parity with the fermion number parity on the superconductor. These predictions still hold even when the metallic ring is disordered and carries more than a single channel. This can be seen in Eq. (3), which remains valid regardless of the microscopic Hamiltonian giving the eigenvalue $\epsilon_{M}$. Impurities can also exist inside the superconductor, in which case they can generate nontopological domains with Majorana bounds states at the domain walls. ${ }^{38,39}$ This can be modeled by replacing the single Majorana site in the effective Hamiltonian of Eq. (1) with a chain of $p$ Majorana sites connected by disordered normal-metallic segments. It is then easy to show using a gauge transformation on the fermionic operators $c$ and $f$ that the $h / e$ harmonic of the persistent current becomes $\sim \sin \left[2 \pi(2 n+1) \phi / \phi_{0}-\chi\right]$ with $\chi=(-1)^{n_{\text {tot }}} p \pi / 2$. Therefore, the general form of Eq. (3) is preserved when there is an odd number of Majorana carrying segments in the chain, while cos $\leftrightarrow$ sin when $p$ is even. Either way, the $h / e$ harmonic persists and changes parity with the parity of the sum of the number of fermions on all Majorana segments.

The persistent current is given by $I(\phi)=-\partial_{\phi} \mathcal{F}$ with the free energy $\mathcal{F}$. In the absence of magnetization, the latter must be an even function of magnetic field, therefore one would expect $I(\phi=0)=0$. The finite zero-flux current predicted above comes about because creating a Majorana state requires one to break time-reversal symmetry with a Zeeman magnetic field in the first place and the free energy is even in the total 
field $\mathcal{F}\left(\phi, B_{\mathrm{Z}}\right)=\mathcal{F}\left(-\phi,-B_{\mathrm{Z}}\right)$. To show that this is the case, we consider the standard nanowire Hamiltonian for Majorana bound states 9,10

$$
H=\left(p_{y}^{2} / 2 m-\mu\right) \tau_{z}+u p_{y} \sigma_{z} \tau_{z}+B_{Z} \sigma_{x}+\Delta \tau_{x} .
$$

The wire is aligned in the $y$ direction and $\sigma_{\alpha}$ and $\tau_{\alpha}$ are Pauli matrices in spin and particle-hole space, respectively. Inverting $B_{\mathrm{Z}}$ is equivalent to space inversion $y \rightarrow-y, p_{y} \rightarrow-p_{y}$, $\sigma_{y} \rightarrow \sigma_{y}, \sigma_{x, z} \rightarrow-\sigma_{x, z}$, and $\tau_{\alpha} \rightarrow \tau_{\alpha}$. Therefore, $B_{Z} \rightarrow-B_{Z}$ is equivalent to interchanging the Majorana operators. This is equivalent to the substitution $\lambda_{1} c_{1} \leftrightarrow \lambda_{2} c_{N}$ in our tight-binding formulation of Eq. (1), which can be absorbed by relabeling the ring operators $c_{i} \rightarrow c_{N-i+1}$ together with $\phi \rightarrow-\phi$ (because the relabeling inverts the direction of counting sites along the ring). Thus the free energy is even $F\left(\phi, B_{\mathrm{Z}}\right)=F\left(-\phi,-B_{\mathrm{Z}}\right)$ and the persistent current is odd in the total magnetic field, as it should be.

It is interesting to note that these symmetry considerations translate into an apparent violation of the Onsager reciprocity relation $G(\phi) \neq G(-\phi)$ for the conductance of the system when it is connected to two external leads. Such an apparent violation was reported in Ref. 16, but its origin was not discussed. The above argument for the symmetry of the free energy can be applied to the transport problem, giving the true Onsager symmetry $G\left(\phi, B_{\mathrm{Z}}\right)=G\left(-\phi,-B_{\mathrm{Z}}\right)$, containing both the Aharonov-Bohm flux and the time-reversal symmetrybreaking field generating the Majorana states in the first place. Such reciprocity relations cannot be violated in two-terminal systems in the linear response regime. Simultaneously, the oscillating part of $G(\phi)$ changes sign each time an electron is added on the superconductor, which agrees with the Friedel sum rule, ${ }^{37}$ according to which the scattering phase jumps by $\pi$ each time the energy of the scattering particle crosses a quasibound state of the scatterer. Topological superconductors therefore present a unique opportunity to verify this sum rule in the presence of superconductivity. In the topologically trivial regime, only Cooper pairs can be added, which results in unnoticeable scattering phase jumps of $2 \pi$.

We finally comment on the short segment limit, when the induced superconducting coherence length is no longer negligible against the length of the superconducting segment. Two additional terms need to be taken into account. First, there is a coupling term between the two Majorana states that is proportional to $\gamma_{1} \gamma_{2}=-i(-1)^{n_{0}}$. That term can be incorporated into the $\delta$ term in the Hamiltonian of Eq. (1) without affecting any of our conclusions. Second, hopping can occur from either side of the ring to either Majorana state. This also can be incorporated into our theory via the substitutions $\lambda_{1} \rightarrow \Lambda_{1}=\lambda_{1}-i \lambda_{1}^{\prime}(-1)^{f^{\dagger} f}$ and $-i \lambda_{2}(-1)^{f^{\dagger} f} \rightarrow$ $\Lambda_{2}=\lambda_{2}^{\prime}-i \lambda_{2}(-1)^{f^{\dagger} f}$ in Eq. (1). The new couplings $\lambda_{1,2}^{\prime}$ are given by overlaps between the left (right) Majorana state and the ring states on the right (left) side of the TS junction. They do not change the harmonic content of the current; moreover, the current does not vanish at zero flux unless $\operatorname{Im}\left(\Lambda_{1}^{*} \Lambda_{2}\right)=0$, which generically does not occur. Furthermore, the sign of the zero-flux current is still determined by the fermion parity. Thus the three signatures of Majorana fermions we predict remain valid even in the limit of a short segment.

We thank C. Beenakker, B. Sothmann, and M. Wimmer for interesting discussions. P.J. thanks the Lorentz Institute, Leiden University and the Department of Theoretical Physics, University of Geneva for their hospitality at the early stage of this project. Research in Geneva was supported by the Swiss NSF, the NCCR MaNEP, and QSIT.

\footnotetext{
${ }^{*}$ Deceased.

${ }^{1}$ V. Mourik, K. Zuo, S. M. Frolov, S. R. Plissard, E. P. A. M. Bakkers, and L. P. Kouwenhoven, Science 336, 1003 (2012).

${ }^{2}$ M. T. Deng, C. L. Yu, G. Y. Huang, M. Larsson, P. Caroff, and H. Q. $\mathrm{Xu}$, Nano Lett. 12, 6414 (2012).

${ }^{3}$ A. Das, Y. Ronen, Y. Most, Y. Oreg, M. Heiblum, and H. Shtrikman, Nat. Phys. 8, 887 (2012).

${ }^{4}$ A. D. K. Finck, D. J. Van Harlingen, P. K. Mohseni, K. Jung, and X. Li, Phys. Rev. Lett. 110, 126406 (2013).

${ }^{5}$ H. O. H. Churchill, V. Fatemi, K. Grove-Rasmussen, M. T. Deng, P. Caroff, H. Q. Xu, and C. M. Marcus, Phys. Rev. B 87, 241401(R) (2013).

${ }^{6}$ L. P. Rokhinson, X. Lui, and J. K. Furdyna, Nat. Phys. 8, 795 (2012). ${ }^{7}$ For reviews on Majorana fermions in a condensed matter context, see J. Alicea, Rep. Prog. Phys. 75, 076501 (2012); C. W. J. Beenakker, Annu. Rev. Condens. Matter Phys. 4, 113 (2013).

${ }^{8}$ A. Y. Kitaev, Phys. Usp. 44, 131 (2001).

${ }^{9}$ R. M. Lutchyn, J. D. Sau, and S. Das Sarma, Phys. Rev. Lett. 105, 077001 (2010).

${ }^{10}$ Y. Oreg, G. Refael, and F. von Oppen, Phys. Rev. Lett. 105, 177002 (2010).

${ }^{11}$ K. T. Law, P. A. Lee, and T. K. Ng, Phys. Rev. Lett. 103, 237001 (2009).
}

${ }^{12}$ A. R. Akhmerov, J. P. Dahlhaus, F. Hassler, M. Wimmer, and C. W. J. Beenakker, Phys. Rev. Lett. 106, 057001 (2011).

${ }^{13}$ L. Fu and C. L. Kane, Phys. Rev. B 79, 161408 (2009).

${ }^{14}$ F. Pietka, A. Romito, M. Duckheim, Y. Oreg, and F. von Oppen, New J. Phys. 15, 025001 (2013).

${ }^{15}$ L. Fu and C. L. Kane, Phys. Rev. Lett. 102, 216403 (2009).

${ }^{16}$ C. Benjamin and J. K. Pachos, Phys. Rev. B 81, 085101 (2010).

${ }^{17}$ J. Li, G. Fleury, and M. Büttiker, Phys. Rev. B 85, 125440 (2012).

${ }^{18}$ D. Bagrets and A. Altland, Phys. Rev. Lett. 109, 227005 (2012).

${ }^{19}$ J. Liu, A. C. Potter, K. T. Law, and P. A. Lee, Phys. Rev. Lett. 109, 267002 (2012)

${ }^{20}$ D. I. Pikulin, J. P. Dalhaus, M. Wimmer, H. Schomerus, and C. W. J. Beenakker, New J. Phys. 14, 125011 (2012).

${ }^{21}$ D. Rainis, L. Trifunovic, J. Klinovaja, and D. Loss, Phys. Rev. B 87, 024515 (2013)

${ }^{22}$ P. San-Jose, E. Prada, and R. Aguado, Phys. Rev. Lett. 108, 257001 (2012).

${ }^{23}$ J. D. Sau, E. Berg, and B. I. Halperin, arXiv:1206.4596.

${ }^{24}$ M. Franz, Nat. Nanotechnol. 8, 149 (2013).

${ }^{25}$ J. Nilsson, A. R. Akhmerov, and C. W. J. Beenakker, Phys. Rev. Lett. 101, 120403 (2008). 
${ }^{26}$ L. Fu, Phys. Rev. Lett. 104, 056402 (2010).

${ }^{27}$ L. P. Lévy, G. Dolan, J. Dunsmuir, and H. Bouchiat, Phys. Rev. Lett. 64, 2074 (1990).

${ }^{28}$ V. Chandrasekhar, R. A. Webb, M. J. Brady, M. B. Ketchen, W. J. Gallagher, and A. Kleinsasser, Phys. Rev. Lett. 67, 3578 (1991).

${ }^{29}$ D. Mailly, C. Chapelier, and A. Benoit, Phys. Rev. Lett. 70, 2020 (1993).

${ }^{30}$ H. Bluhm, N. C. Koshnick, J. A. Bert, M. E. Huber, and K. A. Moler, Phys. Rev. Lett. 102, 136802 (2009).

${ }^{31}$ A. C. Bleszynski-Jayich, W. E. Shanks, B. Peaudecerf, E. Ginossar, F. von Oppen, L. Glazman, and J. G. E. Harris, Science 326, 272 (2009).
${ }^{32}$ M. Büttiker and T. M. Klapwijk, Phys. Rev. B 33, 5114 (1986).

${ }^{33}$ A. A. Reynoso, G. Usaj, C. A. Balseiro, D. Feinberg, and M. Avignon, Phys. Rev. Lett. 101, 107001 (2008).

${ }^{34}$ A. Zazunov, R. Egger, T. Jonckheere, and T. Martin, Phys. Rev. Lett. 103, 147004 (2009).

${ }^{35}$ A. Brunetti, A. Zazunov, A. Kundu, and R. Egger, Phys. Rev. B 88, 144515 (2013).

${ }^{36}$ M. Büttiker and C. A. Stafford, Phys. Rev. Lett. 76, 495 (1996).

${ }^{37}$ J. Friedel, Philos. Mag. 43, 153 (1952).

${ }^{38}$ V. Shivamoggi, G. Refael, and J. E. Moore, Phys. Rev. B 82, 041405 (2010).

${ }^{39}$ K. Flensberg, Phys. Rev. B 82, 180516 (2010). 